\title{
Study of The Effect of Nutrition and Glycemic Control and on IGF-1 and Growth in Pre-pubertal Egyptian Children with T1DM \\ Raef Malak Botros ${ }^{1}$, Aliaa Ahmed Abdo El-Sherbeeny ${ }^{1}$, Nancy Samir El Barbary ${ }^{2}$, Ala Muhammed Muhammed ${ }^{1}$, Hanan Mahmoud Ali ${ }^{* 1}$ \\ Departments of ${ }^{1}$ Internal Medicine, Endocrinology \& Metabolism and ${ }^{2}$ Pediatrics, Faculty of Medicine - Ain Shams University \\ *Corresponding author: Hanan Mahmoud Ali, Mobile: (+20)1222521314, Email: hanan.mahmoud29@yahoo.com
}

\begin{abstract}
Background: Pre-Pubertal children who have T1DM are shorter than their non-diabetic peers.

Objective: In the current study we aimed to evaluate the role of HbA1c and IGF-1 in these cases.

Patients and Methods: The present study was a cross-sectional study conducted on 80 pre-pubertal Egyptian children: 30 males, 30 females with T1DM and 20 age-matched controls (10 males and 10 females), the participants were recruited from the Outpatient Clinic of the Pediatric Department of Ain Shams University Hospitals and the National Institute of Diabetes and Endocrinology in Cairo, Egypt during the period from July 2018 to January 2019.

Results: The height and weight percentiles were found to be lower in type 1 diabetic children when compared to the controls with median values of 14.50 (IQR 27.98) in male patients, 17.95 (IQR 29.18) in female patients, and 87 (IQR 20.58) in the controls. A negative correlation between serum IGF-1 and HbA1c as well as the height and weight percentiles were found with $(\mathrm{P}=0.014,0.009$, and 0.05 respectively). Serum IGF-1 levels were also found to be significantly lower in patients $(\mathrm{P}<0.001)$ with a mean value of $(77.60 \pm 69.377)$ in male patients and $(64.0 \pm$ $29.402)$ in female patients and $(201.0 \pm 102.798)$ in the controls. HbA1c was also found to be negatively correlated with height percentile $(\mathrm{P}=0.012)$.

Conclusion: The glycemic control tested by HbA1c is an important indicator of linear growth in pre-pubertal children, Height, and weight percentiles were found to be lower in type 1 diabetic patients when compared to the healthy controls $(\mathrm{P}<0.001)$ in both cases.
\end{abstract}

Keywords: T1DM, IGF-1, Growth.

\section{INTRODUCTION}

T1DM is considered one of the most common chronic endocrine disorders of childhood ${ }^{(1)}$. The incidence of T1DM in Egypt is found to be 8-10 per 100,000 population per year in children aged less than $15^{(2)}$.

In children and adolescents diagnosed with T1DM, the most frequent complications in hypoglycemia, hyperglycemia, diabetic ketoacidosis (DKA), and psychiatric disorders. The pathogenesis of long-term vascular sequelae including retinopathy, nephropathy, neuropathy, and cardiovascular disease usually starts in childhood, however, the clinical manifestations of these complications are not common before adulthood.

Inadequate growth is also a well-known complication of diabetes. As long as diabetes is known to adversely affect linear growth and pubertal development, so maintenance of good glycemic control in the pre-pubertal diabetic patient is a must to ensure normal physical and psychological growth. Several Studies revealed that growth velocity is affected following the patient's age, also with the age at diagnosis of diabetes mellitus (DM). There is evidence to suggest that growth disorder occurs secondary to abnormalities in the hypothalamicpituitary-growth hormone axis in diabetic patients and these are more common especially in those with poor glycemic control and longer duration of the disease ${ }^{(\mathbf{3})}$.

Growth hormone $(\mathrm{GH})$ secreted in a pulsatile manner with age-dependent concentrations. Most of the $\mathrm{GH}$ promoting growth effects are mediated through the actions of peptides, like insulin-like growth factor I (IGF-1).

IGF-1 is a hormone, structurally similar to insulin. It has an important role in childhood growth. Subjects with T1DM may have low IGF-1 levels, related to insulinopenia and insulin resistance ${ }^{(4)}$.

There is one study that suggested that IGF-1 increased with insulin therapy and better glycemic control $^{(5)}$.

Others concluded that an insulinopenic state has effects on the regulation of IGF-1 similar to those of nutritional deprivation ${ }^{(\boldsymbol{6})}$.

To evaluate the effect of glycemic control on IGF1 and growth, represented by height and weight percentiles for age in a sample of pre-pubertal Egyptian children diagnosed with T1DM.

\section{PATIENTS AND METHODS}

This is a cross-sectional that has been performed during the period from July 2018 to January 2019, conducted on 80 pre-pubertal Egyptian children, 60 of those children were diagnosed with T1DM with 
disease duration more than 4 years, aged 12 or less, and 20 Controls free from the disease, the subjects were recruited from the Outpatient Clinic of the Pediatric Department of Ain Shams University Hospitals and the National Institute of Diabetes and Endocrinology in Cairo, Egypt, the participants were divided into 3 groups: Group I: 30 Males with T1DM, Group II: 30 Females with T1DM, Group III: 20 controls free from DM (10 Males and 10 Females), the purpose of the study was explained to all the participants and their caregivers and consents were obtained. We exclude any Pre-pubertal children with chronic diseases other than T1DM as renal or hepatic disease, Children with thyroid disturbances, Children with a Pituitary disease which was excluded by the history of exposure to Surgery, Trauma, or Irradiation.

Ethical approval and written informed consent: An approval of the study was obtained from Ain Shams University academic and ethical committee. Every patient signed an informed written consent for acceptance of the operation.

\section{All participants were subjected to the following:}

1. Full medical history, one day recall nutritional questionnaire, General clinical examination including blood pressure and pulse, Anthropometric measures: weight, height, skinfold thickness, and mid-arm circumference.

2. Full personal history taking with special emphasis on:

- Name, age, date of birth, and date of interview.

- Dietary history including a 1 day recalls nutritional questionnaire.

- Insulin regimen and Total daily insulin.

- 7-point blood glucose profile from the patient logbook.

\section{Quantitative daily consumption (24-hour nutritional recall) \\ Method:}

1 Day recall nutrition questionnaire:

A questionnaire was utilized to elicit information as shown:

- Can you recall what you ate for breakfast yesterday and the exact amount of each type of food?

- Was there any snack that you had eaten after breakfast and before lunch yesterday?

- Can you recall what you ate for lunch yesterday and the exact amount of each type of food?

- Was there any snack that you have after lunch and before dinner yesterday?

- Can you recall what you ate for dinner yesterday and the exact amount of each type of food?

All the dietary information was collected in detail and the total caloric intake, total daily carbohydrates, total daily proteins, and total daily fats were calculated using the dietary guidelines of the national institute of nutrition in Cairo, Egypt.

\section{Physical Examination:}

Anthropometric measurements, including weight and height, were performed. Standing height using a stadiometer (Seca, Germany), Weight was measured using a digital weighing scale. Height and weight for age percentiles were calculated using CDC height and weight percentile calculators. Centre for Disease Control and Prevention (CDC). National Health and Nutrition Examination Survey, 2008 (http//www.cdc.gov/ growth charts. com). measures of Skinfold thickness were also used to estimate body composition, Triceps Skinfold thickness was measured to the nearest $0.1 \mathrm{~mm}$, using a skinfold caliper, the measurement was taken halfway between the acromion process and the olecranon process. Mid upper- arm circumference (MUAC) has been proposed as another important indicator of obesity in children. Measurements of MUAC have long been known to reflect changes in human body weight, and the major determinants of MUAC, are arm muscle and subcutaneous fat ${ }^{(7)}$.

4. Laboratory investigations: Hemoglobin level, Serum Creatinine, AST-ALT, TSH, Free T4, FBS and 2-hour PP blood sugar, HbA1c, Serum IGF-1 using ELIZA (Enzyme-linked immune-sorbent assay) ${ }^{(\mathbf{8})}$.

\section{Statistical methodology:}

Statistical presentation and analysis of the current study were conducted, using the mean, standard deviation, student t-test, and Chi-square using the SPSS statistical program. Mann-Whitney test: This test was used to analyze height and weight percentiles. ANOVA testing was used for comparison among different times in the same group in quantitative data. P-value $>0.05$ Non-significant, P-value $\leq 0.05$ Significant, P-value < 0.01 Highly Significant. Tuckey testing.

\section{RESULTS}

The current study is a cross-sectional conducted on 80 pre-pubertal Egyptian children divided into 3 groups:

- Group I: 30 Males with T1DM.

- Group II: 30 Females with T1DM.

- Group III: 20 controls free from DM (10 Males and 10 Females).

The current study showed that there is a significant statistical difference between the studied groups regarding the weight, skinfold thickness, and mid-arm circumference $(\mathrm{P}=0.026, \mathrm{P}=0.023, \mathrm{P}=0.006)$ respectively being highest in group III.

As shown in table (1) also there is a significant statistical difference between the studied groups as regards height and weight percentiles $(\mathrm{P}<0.001)$ being highest in group III. As shown in table (2)

On comparing the nutritional status among the studied groups we found that there is a significant difference between the studied groups as regards the 
total daily carbohydrate intake $(P=0.015)$, where the group I was the highest with a mean value \pm SD of $(251.228 \pm 77.068)$ followed by group III (221.03 \pm $14.28)$ then group II $(214.16 \pm 26.246)$. As shown in table (3)

The current study found that there is a highly significant difference between the studied groups as regards the fasting blood sugar $(\mathrm{P}<0.001)$, being highest in group II with a mean value \pm SD of (198.46 $\pm 75.734)$ followed by group I $(176.57 \pm 57.67)$ and lastly group III (92.700 \pm 4.143$)$. Also, there is a highly significant difference between the groups as regards the 2-hour postprandial blood sugar $(\mathrm{P}>0.001)$ being highest in group II $(265.13 \pm 105.43)$ followed by group I (244.10 \pm 58.92$)$ and then group III

$(131.30 \pm 16.017)$, HbA1c had a significant difference between the studied groups $(\mathrm{P}<0.001)$ with group I being the highest $(9.643 \pm 1.485)$ followed by group II $(9.190 \pm 1.125)$ and then group III (4.690 \pm
0.213), Lastly there was a highly significant difference between the three groups as regards the S.IGF-1 levels $(\mathrm{P}<0.001)$ where the level in group III $(201.0 \pm$ 102.79) was higher than both group I (77.60 \pm 69.38$)$ and group II (64.0 \pm 29.40$)$. As shown in table (4)

In the current study we found that there is a positive correlation between IGF-1 and height percentile $(\mathrm{P}=0.009)$, weight percentile $(\mathrm{P}=0.005)$, skinfold thickness $(\mathrm{P}=0.030)$ and mid-arm circumference $(\mathrm{P}=0.037)$. Also, there is a negative correlation between IGF1 and duration of diabetes $(\mathrm{P}$ $=0.020), \operatorname{HbA} 1 \mathrm{c}(\mathrm{P}=0.014)$ as well as total daily proteins $(P=0.004)$. As shown in table (5)

In the current study we found that there is a negative correlation between $\mathrm{HbA} 1 \mathrm{c}$ and height percentile $(\mathrm{r}=$ $-0.324, \mathrm{P}=0.012)$, and weight percentile $(\mathrm{r}-0.248, \mathrm{P}=$ $0.056)$ as shown in table (6)

Table (1): Comparison between the studied groups as regard anthropometric measurement (weight, height, skinfold thickness, and mid-arm circumference):

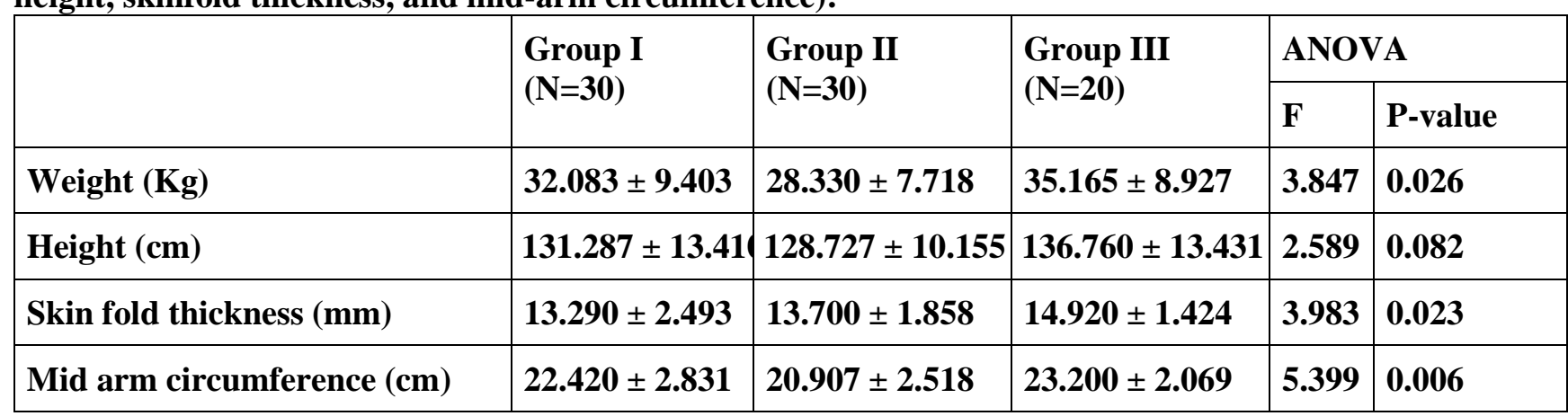

Table (2): Comparison between the studied groups regarding height and weight percentiles for age:

\begin{tabular}{|l|l|l|l|l|l|l|}
\hline \multicolumn{2}{|c|}{} & Group I & Group II & \multirow{2}{*}{ Group III } & \multicolumn{2}{|l|}{ Kruskal -Wallis test } \\
\cline { 4 - 7 } & & & & X & P-value \\
\hline \multirow{2}{*}{$\begin{array}{l}\text { Height } \\
\text { percentile \% }\end{array}$} & Range & $2.8-62.1$ & $0-88.8$ & $67-99.9$ & 42.67 & $<0.001$ \\
\cline { 2 - 5 } & Median (IQR) & $14.50(27.98)$ & $17.95(29.18)$ & $87.00(20.58)$ & & \\
\hline $\begin{array}{l}\text { Weight } \\
\text { percentile \% }\end{array}$ & Range & $2-87.6$ & $1.4-83.6$ & $70.6-99.7$ & 35.05 & $<0.001$ \\
\cline { 2 - 5 } & Median (IQR) & $40.70(41.80)$ & $\mathbf{3 0 . 7 0}(35.60)$ & $\mathbf{8 5 . 4 0}(20.18)$ & & \\
\hline
\end{tabular}

Table (3): Comparison between the studied groups as regard nutritional status:

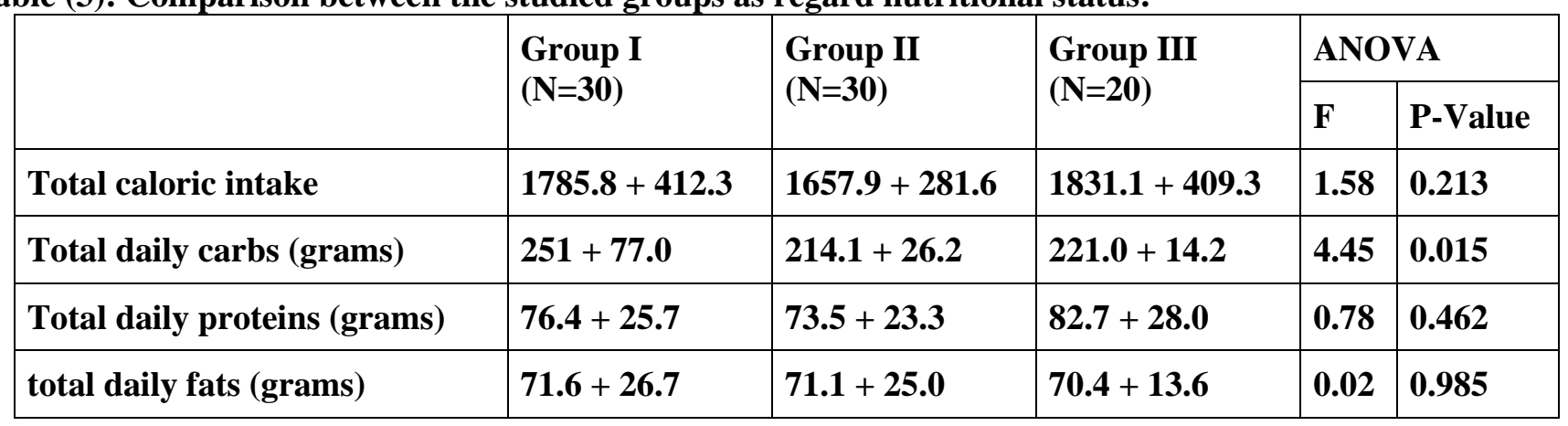


Table (4): Comparison between the studied groups as regard glycemic profile (Fasting blood sugar, 2-hour postprandial blood sugar, HbA1c) and serum IGF1:

\begin{tabular}{|c|c|c|c|c|c|}
\hline \\
\hline & \multirow{2}{*}{$\begin{array}{l}\text { Group I } \\
(\mathrm{N}=\mathbf{3 0})\end{array}$} & \multirow{2}{*}{$\begin{array}{l}\text { Group II } \\
(\mathbf{N}=\mathbf{3 0})\end{array}$} & \multirow{2}{*}{$\begin{array}{l}\text { Group III } \\
(\mathbf{N}=20)\end{array}$} & \multicolumn{2}{|c|}{ ANOVA } \\
\hline & & & & $\mathbf{F}$ & P-Value \\
\hline Fasting blood sugar (mg/dl) & $176.57+57.67$ & $\begin{array}{l}198.47+ \\
75.73\end{array}$ & $92.70+4.14$ & 20.78 & 0.001 \\
\hline $\begin{array}{l}2 \text { hour post prandial blood sugar } \\
(\mathrm{mg} / \mathrm{dl})\end{array}$ & $244.10+58.92$ & $\begin{array}{l}265.13 \\
+105.43\end{array}$ & $131.30+16.01$ & 21.12 & 0.001 \\
\hline HbA1c (\%) & $9.64+1.48$ & $9.19+1.12$ & $4.69+0.21$ & 128.28 & 0.001 \\
\hline S. IGF1 (ng/dl) & $77.60+69.37$ & $64.00+29.40$ & $201.00+102.79$ & 27.08 & 0.001 \\
\hline
\end{tabular}

Table (5): Correlation between anthropometric measures, glycemic profile, nutritional status and duration of diabetes with S.IGF 1:

\begin{tabular}{|l|l|l|}
\hline & \multicolumn{2}{|l|}{ HbA1c } \\
\cline { 2 - 3 } & r & P-value \\
\hline Height percentile (\%) & -0.324 & 0.012 \\
\hline Weight percentile (\%) & -0.248 & 0.056 \\
\hline
\end{tabular}

Table (6): Correlation between height and weight percentile with HbA1c:

\begin{tabular}{|l|l|l|}
\hline & \multicolumn{2}{|l|}{ S.IGF 1 (ng/ dl) } \\
\cline { 2 - 3 } & \multicolumn{1}{|c|}{ r } & \multicolumn{1}{|c|}{ P-value } \\
\hline Height percentile (\%) & 0.334 & 0.009 \\
\hline Weight percentile (\%) & 0.360 & 0.005 \\
\hline Skin fold thickness (mm) & 0.281 & 0.030 \\
\hline Mid arm circumference (cm) & 0.269 & 0.037 \\
\hline FBS (mg/dl) & 0.247 & 0.057 \\
\hline 2hPP (mg/dl) & -0.049 & 0.707 \\
\hline HbA1c (\%) & -0.316 & 0.014 \\
\hline Total caloric intake & 0.171 & 0.190 \\
\hline Total daily carbs (grams) & 0.215 & 0.099 \\
\hline Total daily proteins (grams) & 0.365 & 0.004 \\
\hline Total daily fats (grams) & -0.131 & 0.317 \\
\hline Duration of diabetes & -0.299 & 0.020 \\
\hline
\end{tabular}




\section{DISCUSSION}

The present study revealed that the children with T1DM were significantly shorter and lighter than their non-diabetic peers, this result goes with Khadilkar et al. ${ }^{(3)}$ who found in their study done on 125 diabetic children and age, gender-matched healthy controls, visiting a tertiary care center at Pune, India, All subjects underwent anthropometric measurements (standing height and weight). Diabetes control was evaluated by measuring $\mathrm{HbAlc}$. The results of the study showed that the children with T1DM shorter $(128.3 \pm 24.3 \mathrm{~cm}$ vs. $133.6 \pm 24.7 \mathrm{~cm})$ and lighter $(29.2$ $\mathrm{kg} \pm 15.3$ vs. $31.3 \pm 15.4 \mathrm{~kg}$ ) than the control group.

The current study showed that the level of IGF-1 was significantly lower in the children with T1DM than in the control group, this result came in agreement with Sorensen $\boldsymbol{e t}$ al. ${ }^{(9)}$, who found in a cross-sectional study on 136 prepubertal children and 206 pubertal children/adolescents with T1DM as well as 40 prepubertal and 30 pubertal T1DM free controls for 3-6 years, the study showed that patients with T1DM showed lower IGF-1 levels, when compared to the T1DM free controls.

The current study showed that the poor glycemic control represented by HbA1c levels above 7.5 had a negative impact on the height percentile. Also, the poor glycemic control was found to have a negative impact on IGF-1 in the children with T1DM when compared to their diabetes-free peers, these results are comparable to that of The Wisconsin Diabetes Registry Study, which was an incident cohort study on 45 females and 37 males, followed up from the time of their diagnosis with T1DM for up to 18 years regarding their serum IGF- 1 levels, showed that serum IGF-1 is lower with T1DM than in normative samples. The results of the present study noted a strong association between higher $\mathrm{HbA} 1 \mathrm{c}$ and lower IGF-1 levels, particularly during pre-and early puberty ${ }^{(4)}$.

In the present study, results revealed that there is a negative correlation between S. IGF1 and duration of diabetes, $\mathrm{HbA1c}$, those results are comparable to those of Öberg et al. ${ }^{(10)}$ who found in a cohort study done on 806 children and adolescents with T1DM, where annual blood samples were taken for up to 6 consecutive years. A multiple regression analysis found the IGF-I values to be negatively correlated with HbA1c.

On other hand, this result contrast to results of the study done by Hedman et al. ${ }^{(11)}$, who found in a study done on ten girls and ten boys with T1DM, aged $13.0 \pm 1.4$ (mean $\pm \mathrm{SD}$ ) years at diagnosis, where Blood samples were drawn at the time of diagnosis and after $(3,9,18$, and 48) months to measure HbA1c and IGF-1. The study showed that poor glycemic control was observed at the onset of diabetes and this was associated with low IGF-1. After 3 months, a negative correlation between $\mathrm{HbAlc}$ and IGF-1 was found but not at other time points. In adult type 1 diabetic patients with fair glycemic control, IGF-1 is not associated with $\mathrm{HbA1c}$ while the presence of endogenous insulin secretion influences IGF-1 level independent of glycemic control.

On the other hand, these results do not agree with the results of the study done by Raisingani $\boldsymbol{e t}$ al. (12), who found that the impact of T1DM on growth is no longer of major relevance in developed countries due to more physiological insulin substitutions and self-monitoring. There are also some conflicting reports showing normal or slightly reduced final height in T1DM subjects. In the Oxford study, final heights were not significantly different from the mid-parental height SDS despite a reduced growth spurt during puberty.

As well the results of the present study did not come in line with Giannini et al. ${ }^{(13)}$, who found the relevant role of insulin regimens and in particular of intensive therapy (using four daily insulin injections) from the onset of diabetes in preventing growth alteration in children and adolescents with T1DM has been well defined. In the current study, a group of thirty male children and adolescents with T1DM with different pubertal status (pre-pubertal, pubertal, and post-pubertal) were enrolled and compared with a group of 30 age, sex, matched healthy controls to detect differences in height and serum concentrations of IGF1 and IGFBP-3. Results showed that compared with control subjects IGF-1 and IGFBP-3 serum concentrations were within the normal range in the three diabetic groups, suggesting the normal function of the GH-IGF-1 axis in children with T1DM. There was no significant difference in terms of height SDS documented in the three groups compared with control subjects. Thus, these results suggest that intensive insulin therapy starting from the onset of diabetes might prevent the development of abnormalities of the GH-IGF-I-IGFBP-3 axis, likely allowing normal IGFI and IGFBP-3 levels and physiological growth in children and adolescents with T1DM.

\section{CONCLUSION}

The glycemic control as tested by $\mathrm{HbA1c}$ is an important indicator of linear growth in pre-pubertal children, Height, and weight percentiles were found to be lower in type 1 diabetic patients when compared to healthy controls $(\mathrm{P}<0.001)$ in both cases. Serum IGF1 levels were also found to be much lower in patients with T1DM than in controls $(P<0.001)$. Serum IGF-1 also revealed a significant negative correlation with HbA1c.

\section{Conflict of interest:}

The authors declare no conflict of interests. 
There was no financial funding from any institution. We declare receiving no funds or grants for this study.

\section{REFERENCES}

1. Silverstein $\mathbf{J}$, Klingensmith $\mathbf{G}$, Copeland $\mathrm{K}$ et al. (2005): Care of children and adolescents with type 1 diabetes. Diabetes Care, 28(1): 186-212.

2. El-Ziny M, Salem N, El-Hawary L et al. (2014): Epidemiology of childhood type 1 diabetes mellitus in Nile Delta, northern Egypt-a retrospective study. Journal of Clinical Research in Pediatric Endocrinology, 6 (1): 913.

3. Khadilkar V, Parthasarathy L, Mallade B et al. (2013): Growth status of children and adolescents with type 1 diabetes mellitus. Indian Journal of Endocrinology and Metabolism, 17(6): 1057.

4. Palta M, LeCaire T, Sadek-Badawi M et al. (2014): The trajectory of IGF-1 across age and duration of type 1 diabetes. Diabetes/ Metabolism Research and Reviews, 30(8): 777-783.

5. Bereket A, Lang C, Wilson $T$ (1999): Alterations in the growth hormone-insulin-like growth factor axis in insulin-dependent diabetes mellitus. Hormone and Metabolic Research, 31(02/03): 172-181.

6. Dills D, Allen C, Palta M et al. (1995): Insulin-like growth factor-I is related to glycemic control in children and adolescents with newly diagnosed insulin-dependent diabetes. J Clin Endocrinol Metab., 180:2139-43.

7. Yallamraju S, Mehrotra R, Sinha A et al. (2014): Use of mid-upper arm circumference for evaluation of the nutritional status of OSMF patients. Journal of International Society of Preventive \& Community Dentistry, 4(2): 122-26.

8. Friedrich N, Krebs A, Nauck M et al. (2010): Age-and gender-specific reference ranges for serum insulin-like growth factor I (IGF-I) and IGF-binding protein-3 concentrations on the Immulite 2500: results of the Study of Health in Pomerania (SHIP). Clinical Chemistry and Laboratory Medicine, 48(1): 115-120.

9. Sorensen J, Birkebaek N, Bjerre $M$ et al. (2015): Residual $\beta$-cell function and the insulin-like growth factor system in Danish children and adolescents with type 1 diabetes. The Journal of Clinical Endocrinology \& Metabolism, 100(3): 1053-1061.

10. Öberg D, Salemyr J, Örtqvist E et al. (2018): A longitudinal study of serum insulin- like growth factorI levels over 6 years in a large cohort of children and adolescents with type 1 diabetes mellitus. Pediatr Diabetes, 19(5):972-978.

11. Hedman C, Frystyk J, Lindström $T$ et al. (2004): Residual $\beta$-cell function more than glycemic control determines abnormalities of the insulin-like growth factor system in type 1 diabetes. The Journal of Clinical Endocrinology \& Metabolism, 89(12): 6305- 6309.

12. Raisingani M, Preneet B, Kohn B et al. (2017): Skeletal growth and bone mineral acquisition in type 1 diabetic children, abnormalities of the GH/IGF-1 axis. Growth Hormone \& IGF Research, 34: 13-21.

13. Giannini C, Mohn A, Chiarelli F (2014): Growth abnormalities in children with type 1 diabetes, juvenile chronic arthritis, and asthma. Int J Endocrinol., 2014: 265954 